\title{
Proyección de Rosenberg en la evaluación radiológica de la osteoartrosis de rodilla
}

\author{
Cristián Fontboté $R^{1}$, Fernando Nemtala $U^{1}, 0$ scar \\ Contreras $\mathbf{0}^{2}$, Rosario G uerrero ${ }^{1}$.
}

Rosenberg projection

for the radiological diagnosis of knee osteoarthritis

Background: Knee osteoarthritis is highly prevalent in our country. An early diagnosis is very important to provide an accurate and early treatment, and eventually delay its progression. Aim: To compare the diagnostic yield for osteoarthritis of two projections in knee X ray examination. Material and Methods: We evaluated 44 knees with conventional (AP weight-bearing, in full extension) and Rosenberg (PA weight-bearing in 45 degrees of flexion) X ray projections in 32 patients ( 24 women and 8 men), aged 26 to 78 years. All patients were symptomatic at the time of evaluation, with a suspicion of knee osteoarthritis. Both projections were analyzed and compared. Results: Joint space in the lateral compartment with conventional AP weight-bearing and Rosenberg projections were $8.6 \pm 11.5 \mathrm{~mm}$ and $7.8 \pm 10.9 \mathrm{~mm}$, respectively (NS). The joint space for the medial compartment were $8.2 \pm 10.9 \mathrm{~mm}$ and $6.7 \pm 8.6 \mathrm{~mm}$, respectively ( $\mathrm{p}<0.05$ ). Conclusions: Rosenberg projection is more sensitive to detect knee joint space narrowing as compared with conventional AP weight-bearing projection, at least in the medial compartment of the knee. Therefore, Rosenberg projection should be included in the initial study of any patient with suspicion of knee osteoarthritis (Rev Méd Chile 2008; 136: 880-4).

(Key words: Osteoarthritis, knee; Radiology; X-rays)

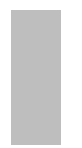

\begin{abstract}
Recibido el 16 de mayo, 2007. Aceptado el 24 de marzo, 2008.
${ }^{1}$ Departamento de Ortopedia y Traumatología, Facultad de Medicina, Pontificia Universidad Católica de Chile; ${ }^{2}$ Departamento de Radiología, Facultad de Medicina, Pontificia Universidad Católica de Chile, Santiago de Chile.

Los autores declaran no haber recibido fuentes de apoyo financiero de ninguna especie para la confección del presente manuscrito.
\end{abstract}

L a osteoartrosis de la rodilla es una patología cuya prevalencia se ha incrementado en las últimas décadas, debido a múltiples factores como el incremento de la expectativa de vida de la población, las altas tasas de obesidad, deportes de impacto, etc.

Correspondencia a: Dr. Cristián Fontboté R. Departamento de Ortopedia y Traumatología, Pontificia Universidad Católica de Chile. Marcoleta 352, Patio Interior (1 ${ }^{\text {er }}$ piso). Santiago. Teléfono: (56-2) 3543467. E mail: cfontbote@med.puc.cl
Además de un examen físico completo y minucioso de la articulación involucrada, los medios de diagnóstico por imágenes tienen un rol muy importante en la confirmación de esta patología.

El primer cambio anatomopatológico que se observa en esta patología es la disminución del grosor del cartílago articular, que se traduce en la radiografía como una disminución del espacio articular.

$\mathrm{Si}$ bien medios más sofisticados como la resonancia magnética permiten estudiar con alta 
precisión los cambios degenerativos articulares, la radiografía simple aún constituye el estudio básico e inicial más importante de esta patología 9 .

Sin embargo, la técnica empleada en la obtención de la radiografía es fundamental para lograr visualizar los cambios que se presentan, tanto en etapas iniciales como en su evolución en el tiempo.

Ya en 1937, E Holmblad demostró que las radiografías tomadas con la rodilla en flexión, aunque sin carga, permitían definir con mayor precisión los cambios degenerativos del surco intercondíleo, espinas tibiales y espacio articular, en comparación con la radiografía antero-posterior (AP) sin carga ${ }^{1}$.

En 1968, Ahlback describió que las radiografías AP de rodilla en extensión y con carga, lograban revelar de manera más real la disminución del espacio articular en comparación con las radiografías tomadas en decúbito y sin carga. Además, demostró que el espacio articular visto en las radiografías podía disminuir aún más si éstas eran tomadas inmediatamente después de que el paciente caminara por algunos minutos, atribuyendo este fenómeno a la adaptación de las superficies articulares irregulares ${ }^{2}$.

T Marklund y R Myrnerts, en 1974, demostraron que ya se producía una reducción del espacio articular tibiofemoral con tan sólo 10 grados de flexión en la proyección AP con carga comparada con la proyección AP convencionall1.

Mediante técnicas de artroscopia se ha demostrado que la pérdida del cartílago articular sigue, por lo general, un patrón asimétrico produciéndose un mayor desgaste inicial en la zona de carga del cartílago articular de los cóndilos femorales entre 30 y 60 grados. Biomecánicamente, esto se debe a que durante la marcha la superficie de carga disminuye al máximo con cierto grado de flexión, desgastando de forma más precoz la región posterior de los cóndilos, por lo que las radiografías en extensión aunque con carga podrían subvalorar cambios tempranos en la altura del espacio articular?

D Resnick y V Vint, en 1980, estudiaron 12 pacientes con gonartrosis, encontrando que la proyección AP con carga en 45 grados de flexión también llamada "proyección del túnel" permitía una cuantificación más precisa de la altura del cartílago articular en comparación con radiografías AP con carga con la rodilla en extensión ${ }^{3}$.

Otros estudios que compararon los hallazgos radiográficos en la proyección AP convencional con carga con los cambios del tejido condral evidenciados mediante artroscopia, concluyeron que este tipo de proyección tenía una baja especificidad, la que oscilaba entre $50 \%$ y $60 \%$, dependiendo del compartimento evaluado, siendo menor para el compartimento lateral ${ }^{10}$.

También S Messieh et al en un estudio en 64 pacientes con sospecha de gonartrosis, midieron 198 compartimentos femorotibiales con radiografías de rodilla AP con carga en extensión y flexión de 30 grados. Encontraron que esta última era capaz de mostrar una mayor disminución del espacio articular de 2 a 3,2 $\mathrm{mm}^{4}$.

PG Maquet y cols, en un estudio en cadáveres, estudiaron la distribución de las cargas en el cartílago articular tibiofemoral durante los movimientos de flexoextensión y concluyeron que con la rodilla en extensión la superficie de contacto cuya dimensión oscilaba entre 18,22 $\mathrm{cm}^{2}$ y 21,95 $\mathrm{cm}^{2}$ con una media de 20,13 $\mathrm{cm}^{2}$. También observaron que las superficies de carga disminuían hasta un promedio de $11,6 \mathrm{~cm}^{2}$ entre $90 \mathrm{y}$ 110 grados de flexión y que éstas además se desplazaban hacia posterior ${ }^{8}$.

$\mathrm{T}$ Rosenberg, en un estudio en 55 pacientes con diversos grados de gonartrosis comprobados mediante cirugía, comparó la proyección convencional con la rodilla en extensión con carga, con una proyección posteroanterior con carga pero con flexión de 45 grados. La idea de esta nueva proyección se basó en su experiencia quirúrgica artroscópica, tras haber observado que el mayor desgaste condral ocurre entre 30 y 60 grados de flexión, debido a una menor área sometida a una mayor carga. Esta nueva proyección en flexión demostró ser mucho más sensible que las proyecciones convencionales ( $80 \%$ vs $30 \%$ ) en ese estudio ${ }^{5}$.

Muchas veces los especialistas en ortopedia y traumatología recibimos pacientes con sospecha de gonartrosis que son denivados ya con estudios radiológicos por parte de médicos no especialistas, y muchas veces estos estudios no son los más apropiados en cuanto a su utilidad y acusiocidad para conocer el verdadero estado del cartílago articular de rodilla, debiendo repetir los estudios radiológicos, con la pérdida de tiempo, dinero y tiempo que eso conlleva.

El objetivo de este trabajo es comparar en el mismo paciente y en la misma rodilla que sufre la misma patología (osteoartrosis), dos proyecciones radiológicas usadas para estudio de la gonartrosis, 
como son la proyección anteroposterior (AP) con carga en extensión completa y la proyección posteroanterior (PA) con carga en flexión de $45^{\circ}$ (proyección de Rosenberg).

La hipótesis de trabajo es que la proyección PA con carga en flexión (Rosenberg) muestra en mejor forma, más precisa y verdadera, la real disminución del espacio articular en la rodilla que sufre de osteoartrosis, en comparación con la radiografía AP con carga en extensión completa.

\section{PACIENTES Y MÉTODOS}

Durante los meses de junio, julio y agosto de 2003, en el Hospital Clínico de la Universidad Católica de Chile, 44 rodillas en 32 pacientes con sospecha de gonartrosis fueron estudiados mediante 2 proyecciones radiográficas: AP (anteroposterior) con carga en extensión y PA (posteroanterior) con carga en flexión de 45 grados (proyección de Rosenberg).

La edad de los pacientes tuvo una mediana de 61 años, con un rango entre 26 y 78 años, con una distribución de $74 \%$ mujeres y 26\% hombres (Tabla 1).

Las radiografías fueron tomadas según la técnica descrita por Rosenberg, con el paciente de pie y el peso distribuido de forma simétrica en las dos extremidades, los ortejos apuntando hacia el frente y la rótula apoyada en la placa de $\mathrm{Rx}$. La rodilla se llevó hasta 45 grados de flexión y el disparador de rayos $\mathrm{X}$ se posicionó a nivel del polo inferior de la rótula, con 10 grados de angulación hacia caudal de modo que los márgenes anterior y posterior de la tibia se sobrepongan (Figura 1). Se utilizó técnica libre para la toma de radiografías, con grilla fija, con método radiológico digital directo (equipo G.E. Proteus), con 65 KVP y 50 MAS, y una distancia foco-película de $100 \mathrm{~cm}$.

Todas las radiografías fueron certificadas técnicamente correctas y fueron medidas (espacio

Tabla 1. C aracterísticas de los pacientes

\begin{tabular}{|llr|}
\hline N =32 pacientes / 44 rodillas & \\
Edad (años) & Mediana & 61 \\
& Rango & $26-78$ \\
Género (\%) & Masculino & 26 \\
& Femenino & 74 \\
\hline
\end{tabular}

articular), por un único radiólogo especialista en patología osteoarticular de nuestra institución.

En ambas proyecciones se midieron los espacios articulares más estrechos en los compartimentos medial y lateral tibio femoral.

Se calculó un tamaño muestral mínimo de 25 pacientes para proveer un poder estadístico de $80 \%$, para detectar una diferencia de $1,7 \pm 3,0 \mathrm{~mm}$ en la medición del espacio articular, con un emor tipo I de 0,05 . La diferencia de $1,7 \mathrm{~mm}$ es especulativa, basada en el trabajo original de Rosenberg $\mathrm{T}$ et $\mathrm{al}^{5}$, que definieron como un estrechamiento del espacio articular a una disminución igual o mayor a 2,0 mm en el compartimento tibiofemoral estudiado, lo que traduce un desgaste en el cartílago articular significativo ${ }^{5}$.

Para el análisis de los datos se utilizó el test Chi cuadrado para la comparación de porcentajes, el test $t$ de Student para variables paramétricas y el análisis de la varianza (ANOVA) para la comparación de promedios de medidas repetidas.

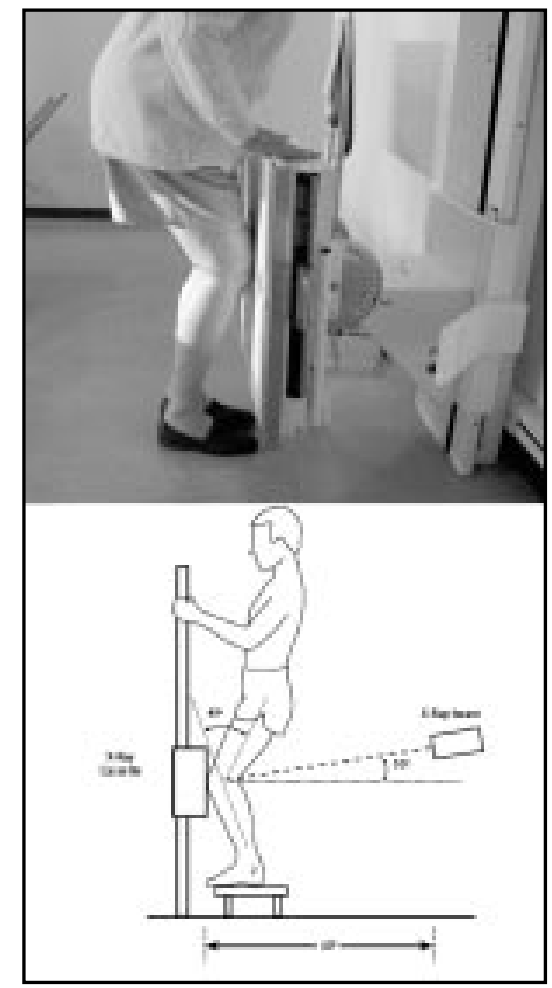

Figura 1. Técnica para la toma de la proyección de Rosenberg. La rótula apoyada en la placa de Rx. La rodilla se lleva hasta 45 grados de flexión y el haz de rayos $\mathrm{X}$ se posiciona a nivel del polo inferior de la rótula, con 10 grados de angulación hacia caudal. 
Se calculó el valor promedio del espacio articular en milímetros de cada compartimiento en ambas proyecciones radiográficas y posteriormente fueron comparados.

\section{RESULTADOS}

Para el compartimiento lateral, el promedio de espacio articular fue de $8,6 \pm 11,5 \mathrm{~mm}$ en la proyección AP y de 7,8 $\pm 10,9 \mathrm{~mm}$ en la proyección de Rosenberg, diferencia que no fue estadísticamente significativa.

Para el compartimiento medial el promedio en la proyección AP fue de $8,2 \pm 10,9 \mathrm{~mm}$ y de $6,7 \pm 8,6$ $\mathrm{mm}$ en la proyección de Rosenberg, diferencia estadísticamente significativa ( $p<0,05)$.

Para el compartimiento lateral, la diferencia promedio AP vs Rosenberg fue de $0,82 \pm 6,3 \mathrm{~mm}$, diferencia que no fue estadísticamente significativa $(p=0,104)$.

Para el compartimiento medial, la diferencia promedio AP vs Rosenberg fue de $1,5 \pm 3,5 \mathrm{~mm}$, diferencia que fue estadísticamente significativa $(<0,05)$ (Tabla 2).

Se pudo observar que la proyección de Rosenberg en comparación con la proyección AP tiene mayor capacidad de detectar la disminución del espacio articular cuanto mayor es este (más espacio remanente), es decir la proyección de Rosenberg, a diferencia de la proyección AP convencional, es capaz de detectar estadios de artrosis incipiente. A medida que la enfermedad progresa y existe una disminución considerable en la altura del espacio articular, ambas proyecciones tienen un rendimiento similar (Figuras 2A y 2B).

\section{DisCUSIÓN}

La disminución del espacio articular es el primer signo radiológico de la gonartrosis, es por eso que se requiere un método de estudio que permita estudiar este fenómeno en su real magnitud y con

Tabla 2. M edición espacio articular

\begin{tabular}{|lccccc|}
\hline Medición espacio articular & $\begin{array}{c}\mathrm{N} \\
\text { pacientes/rodillas }\end{array}$ & $\begin{array}{c}\text { AP convencional } \\
(\mathrm{mm})\end{array}$ & $\begin{array}{c}\text { Rosenberg } \\
(\mathrm{mm})\end{array}$ & $\begin{array}{c}\text { Delta } \\
(\mathrm{mm})\end{array}$ & $\mathrm{p}$ \\
\hline Femorotibial medial & $30 / 44$ & $8,2 \pm 10,9$ & $6,7 \pm 8,6$ & $1,5 \pm 3,5$ & $<0,05$ \\
Femorotibial lateral & $30 / 44$ & $8,6 \pm 11,5$ & $7,8 \pm 10,9$ & $0,82 \pm 6,3$ & 0,104 \\
\hline
\end{tabular}

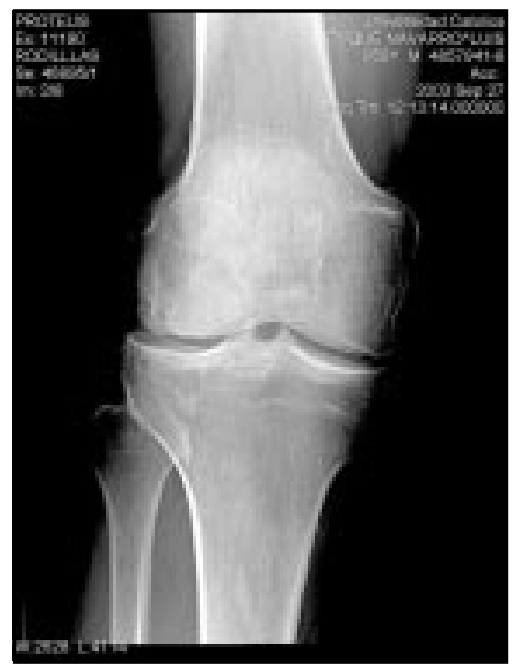

Figura 2A. Proyección radiográfica AP convencional de un paciente con síntomas de gonartrosis. Los espacios articulares se ven conservados.

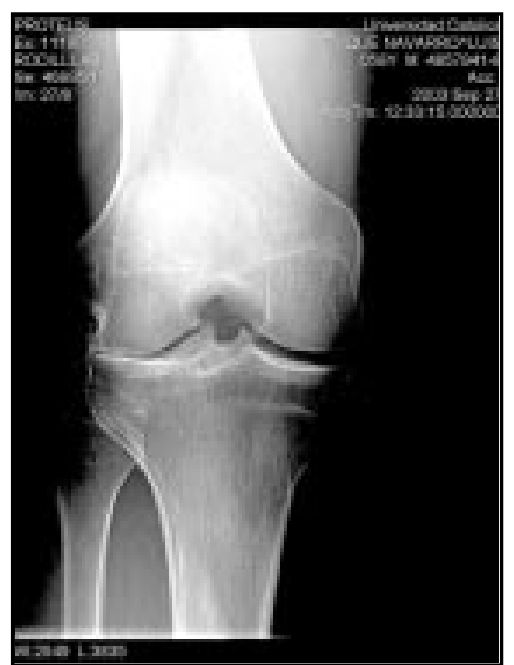

Figura 2B. Proyección de Rosenberg del mismo paciente, una importante disminución del espacio tibiofemoral lateral. 
la mayor sensibilidad en la detección precoz de este fenómeno para orientar al tratante en las guías de tratamiento con este grupo de pacientes.

Son varios los trabajos que respaldan las ventajas de la proyección en flexión con carga en el estudio radiográfico de la gonartrosis, por sobre la proyección AP en extensión.

Durante la marcha, las presiones intrarticulares oscilan entre 3 y $19 \mathrm{Kg} / \mathrm{cm}^{2}$ y el área de contacto entre las superficies articulares varía de 17 y 20 $\mathrm{cm}^{2}$, siendo estas últimas menores con la flexión. De este modo, el cartílago articular sufre un desgaste irregular, siendo mayor en la zona posterior de los cóndilos femorales, cuando la rodilla se encuentra entre 30 y 60 grados de flexión.

Varios autores han reportado las ventajas de las radiografías con distintos ángulos de flexión, sin embargo la proyección de Rosenberg muestra el espacio articular en la zona más representativa de daño condral, permitiendo evaluar de manera más fidedigna los cambios en su altura (conservación 0 disminución del grosor del cartílago articular).

Con la proyección AP convencional, al estar la rodilla en extensión, muestra la altura del cartílago articular de la región anterior de los cóndilos que suele estar poco alterada, es decir, no refleja el desgaste articular precoz que se produce más posterior en los cóndilos femorales, lo que sí se visualiza con la radiografía en flexión.

La proyección de Rosenberg adicionalmente ofrece una visión del surco intercondíleo, las

\section{REFERENCIAS}

1. Holmblad E. Postero-Anterior $X$ ray view of the knee in flexion. J Am Med Assn 1937; 109: 1196-7.

2. Анцваск S. Osteoarthrosis of the knee. A Radiographic Investigation. Acta Radiol 1968; Suplemento 277: 7-72.

3. Resnick D, Vint V. The "Tunnel" view in assessment of cartilage loss in osteoarthritis of the knee. Radiology 1980; 137: 547-8.

4. MESSIEH S. Anteroposterior radiographs of the osteoarthritic knee. J Bone Joint Surg Br 1990; 72-B: 639-40.

5. Rosenberg T, Paulos L, Parker R, Coward D, Scott S. The forty-five-degree posteroanterior flexion weight-bearing radiograph of the knee. J Bone Joint Surg Am 1988; 70-A: 1479-83.

6. Bland J, Altman D. Statistical methods for assessing agreement between two methods of clinical measurement. Lancet 1986; 1: 307-10. espinas tibiales y sería capaz de demostrar condiciones patológicas tales como osteofitos, cuerpos libres intrarticulares, osteocondritis disecante y osteonecrosis de los cóndilos femorales, cuyo estudio en las radiografías convencionales AP en extensión es menos sensible.

En este trabajo comprobamos que la proyección de Rosenberg efectivamente brinda una información más acuciosa en el estudio de la artrosis femorotibial, ya que muestra la real disminución del espacio articular y, por lo tanto, el verdadero estado de progresión de la enfermedad artrósica, por lo menos en el espacio femorotibial medial.

Basados en los resultados de este trabajo, podemos recomendar que la proyección de Rosenberg debe ser la de elección en pacientes con sospecha de gonartrosis, especialmente en el compartimiento tibio femoral medial, por ejemplo, en pacientes con genu varo.

En este estudio de serie de casos no se utilizó un grupo control, ya que el objetivo es la comparación de dos métodos de estudio radiológico para una misma patología (osteoartrosis de rodilla), por lo que el estudio de rodillas sin patología no fue incluido.

Basados en este trabajo de investigación clínica, cuyo grupo poblacional fue preferentemente mujeres, podemos sugerir el uso preferente de la proyección de Rosenberg en el estudio de la artrosis de rodilla, especialmente en pacientes de sexo femenino con sospechas de osteoartrosis secundario a genu varo.

7. KetTelKamp D, Jacobs A. Tibiofemoral contact area. Determination and implications. J Bone Joint Surg Am 1972; 54A; 349-56.

8. Maquet P, VAn de Berg A, Simonet J. Femorotibial weight bearing areas. J Bone Joint Surg 1975; 57A: 766-72.

9. Altman R, Asch E, Bloch D, Bole G, Borenstein D, Brandt $K$ ET AL. Development of criteria for the classification and reporting of osteoarthritis. Arthritis and Rheumatism 1986; 8: 1039-49.

10. Fife R, Brandt K, Braunstein E, Katz B, Shelbourne D, KALASINSKI L ET AL. Relationship between arthroscopic evidence of cartilage damage and radiographic evidence of joint space narrowing in early osteoarthritis of the knee. Artritis and Rheumatism 1991; 4: 377-82.

11. MarkLUnd T, Myrnerts R. Radiographic determination of cartilage height in the knee joint. Acta Orthop Scand 1974; 45: 752-5. 